\title{
GABRB3 mutation as a cause of Ohtahara syndrome: A case report
}

\author{
Mohini Bhelo ${ }^{1}$, Harshita Jagwani², Swapan Mukherjee ${ }^{3}$, Hriday De ${ }^{4}$, Apurbo Ghosh ${ }^{5}$ \\ From ${ }^{1}$ Junior Resident, ${ }^{2}$ Senior Registrar, Department of Pediatric Medicine, ${ }^{3}$ Consultant Neurologist, Department of Pediatric Neurology, ${ }^{4}$ Assistant \\ Professor, ${ }^{5}$ Director, Institute of Child Health, Kolkata, West Bengal, India
}

\begin{abstract}
Ohtahara syndrome (OS) is a rare early infantile epileptic encephalopathy that is characterized by an abnormal electroencephalogram (EEG) and intractable seizures. The patient of this reported case is a 4-month-old male infant delivered by cesarean section with an uneventful antenatal and neonatal period. At 2 months of age, he developed seizures that were refractory to anticonvulsants. Prolonged video EEG showed a characteristic suppression-burst pattern. We report an infant OS associated with heterozygous mutation in the GABRB3 gene.
\end{abstract}

Keywords: Ohtahara syndrome, Suppression-burst pattern, GABRB3 mutation

$\mathrm{E}$ arly infantile epileptic encephalopathy (EIEE) or Ohtahara syndrome (OS) is a rare and earliest form of agedependent epileptic encephalopathies with poor prognosis, characterized by an abnormal electroencephalogram (EEG) and intractable seizures in the neonatal and early infantile period [1]. EEG shows a characteristic suppression-burst pattern during both the sleeping and awake states, as well as frequent tonic spasms. It is a progressive neurological disorder associated with delayed motor and cognitive development and intractable seizures [2]. OS is not a homogeneous entity and has multiple presumed etiologies. Although virtually all reported cases of OS are associated with a congenital or acquired structural malformation of cortical development, in our case, there was no structural abnormality.

To contribute to the understanding of this severe early epileptic encephalopathy, we report a clinical neuropathologic case of a 4-month-old infant diagnosed as OS with heterozygous mutation in GARBB3 gene, who presented with intractable seizure along with a characteristic suppression-burst pattern in EEG and no structural abnormality.

\section{CASE REPORT}

A 4-month-old male child, term by gestation, the second child of healthy non-consanguineous parents was delivered by lower uterine cesarean section with a birth weight of $3.2 \mathrm{~kg}$. There was no history of maternal drug use, irradiation, or significant events in the antenatal period. The baby cried immediately after birth with APGAR scores of 8 and 9 at 1 and $5 \mathrm{~min}$, respectively, and

\section{Access this article online}

Received - 23 September 2021

Initial Review - 09 October 2021

Accepted - 29 October 2021

DOI: $10.32677 /$ ijcr.v7i11.3112 was apparently normal till 2 months of age. Family history was non-contributory. The patient presented with failure to thrive in early infancy. At 2 months of his age, he started having clusters of short seizures (each episode persisting approximately $1 \mathrm{~min}$ ) characterized by jerky movements of the bilateral or sometimes unilateral upper limb, eye deviation, and eyelid flickering. There were no dysmorphic features of the face, trunk, or limbs and no evidence of any neurocutaneous lesion. There was no hepatosplenomegaly.

Initially, the child had developed a social smile which disappeared along with loss of suck swallow reflex at 4 months of his age.

Magnetic resonance imaging (3 Tesla magnet, standard acquisition protocol) brain showed no significant findings. Extensive neurometabolic investigations including serum ammonia and lactate were found within normal limits. Amino acid analysis of the blood was also within the normal limit. TORCH screening was negative and cerebrospinal fluid study was within normal limit. Initially, a sleep EEG was done which showed no significant abnormality.

The child was started initially on anticonvulsants phenobarbitone $(3 \mathrm{mg} / \mathrm{kg})$ along with levetiracetam $(20 \mathrm{mg} / \mathrm{kg})$ and the doses were further increased. However, the child continued to have refractory intractable epilepsy despite multiple antiepileptic therapies (levetiracetam $60 \mathrm{mg} / \mathrm{kg} / \mathrm{day}$, phenobarbitone $5 \mathrm{mg} / \mathrm{kg} /$ day, and clobazam $5 \mathrm{mg}$ once daily).

Another $1 \mathrm{~h}$ video EEG was done which showed no significant abnormality. Then, the child was started on biotin, pyridoxine, and folinic acid. A $5 \mathrm{~h}$, video EEG was done which revealed a suppression-burst pattern. A whole-exome sequencing test was done which revealed a heterozygous missense variation in exon

Correspondence to: Hriday De, Dr. Biresh Guha Street, Institute of Child Health, Kolkata - 700 017, West Bengal, India. E-mail- hridayde2010@gmail.com

(C) 2021 Creative Commons Attribution-NonCommercial 4.0 International License (CC BY-NC-ND 4.0). 
7 of the GABRB3 gene (CHR15:G.26567659G>A) that results in the amino acid substitution of serine for proline at codon 253.

The child was then started on clonazepam $(0.03 \mathrm{mg} / \mathrm{kg} / \mathrm{day})$ and was discharged after 2 days with the prognosis being explained to the parents. On follow-up after 1 week, parents reported the frequency of seizures to be decreased but not being fully controlled. Clonazepam dose was then increased to $0.05 \mathrm{mg} / \mathrm{kg} /$ day. However, the child was lost to follow-up after that and we could not trace the course of the illness thereon.

\section{DISCUSSION}

OS popularly known as the EIEE is an electroclinical syndrome occurring within the first 3 months of age [1]. Seizures range from tonic spasms which are independent of the sleep cycle to focal motor seizures, hemiconvulsion, and generalized tonic-clonic seizures [2]. EEG shows a characteristic burst-suppression pattern that coincides with tonic spasms [3].

The majority of OSs have been attributed to structural brain abnormalities including hemimegalencephaly, agenesis of the corpus callosum, porencephaly, agenesis of mammillary bodies, cortical dysplasia, and cerebral migration disorders [4]. Although genetic and metabolic causes have been reported in some cases of OS, there are very few reports describing GABRB3 mutation as a potential cause. Hence, we report the case of a 4-month-old male infant who presented with intractable seizures from 2 months of age and was diagnosed with OS due to a heterozygous mutation in the GARBB3 gene in the absence of a structural brain malformation.

Previously, Pavone et al. reported mutations in four genes causing OS: (a) ARX (aristaless related) homeobox gene at Xp22.13, (b) CDKL15 (SYK9) gene at Xp22, (c) the SLC 25 A 22 (GC1) gene at 11p15.5, and (d) Stxbp1 (MUNC18-1) gene at 9q34-1 [5]. As per a study by Kodera et al., out of 526 patients with infantile epilepsy, the whole-exome sequencing revealed de novo GABRA1 mutation in four patients. In addition, GABRA1 resequencing done in 145 patients showed de novo GABRA1 mutations in two patients [6].

GABRB3 located on chromosome 15q11.2-q12 encodes the B3 subunit of $\mathrm{GABA}^{\mathrm{a}}$ receptor which is a ligand-gated chloride channel mediating inhibitory signals within the central nervous system. Allen et al. reported five cases of GABRB3 mutation causing infantile seizures. All five were de novo GABRB3 mutations. Subsequently, three children were diagnosed to have Lenox Gastaut syndrome. On follow-up, two patients were observed to have attention-deficit hyperactive disorder (ADHD) while one had features of mild autism [7].

The common features observed in GABRB3 mutation causing infantile encephalopathy were seizure onset before 10 months of age, multiple seizure types including infantile spasms, variable
EEG abnormalities associated with neurodevelopmental delay, ADHD, autism, and intellectual disability. Our infant developed multiple seizures below 3 months of age with neurodevelopmental delay. Initially, video EEG performed for a span of $1 \mathrm{~h}$ did not reveal any abnormality. However, video EEG performed for $5 \mathrm{~h}$ revealed a characteristic suppression-burst pattern. Hence, a video EEG for a prolonged period is ideal in infants with epileptic encephalopathy.

The prognosis of OS is poor. Antiepileptic drugs such as benzodiazepine, valproate, levetiracetam, zonisamide, phenobarbitone, and pyridoxine have shown limited success in controlling seizures [2,8]. Although our case did show improvement after the addition of pyridoxine and benzodiazepines, the seizures were yet not fully controlled. Ishi et al. reported an infant who responded well to a ketogenic diet after failed multiple anticonvulsants [9]. Subsequently, these patients may evolve into West syndrome and Lennox-Gastaut syndrome.

\section{CONCLUSION}

GABRB3 mutation is a recently described genetic cause of EIEE. Although neuroimaging may be found normal in such cases, a genetic diagnosis should always be sought for.

\section{REFERENCES}

1. Ohtahara S. Seizure disorders in infancy and childhood. Brain Dev 1984;6:509e-19.

2. Yamatogi Y, Ohtahara S. Early-infantile epileptic encephalopathy with suppression-bursts, ohtahara syndrome: Its overview refer-ring to our 16 cases. Brain Dev 2002;24:13e-23.

3. Fusco L, Pachatz C, Di Capua M, Vigevano F. Video-EEG aspects of early-infantile epileptic encephalopathy with suppression-bursts (ohtahara syndrome). Brain Dev 2001;23:708e-14.

4. Miller SP, Dilenge ME, Meagher-Villemure K, O’Gorman AM, Shevell MI. Infantile epileptic encephalopathy (ohtahara syndrome) and migrational disorder. Pediatr Neurol 1998;19:50-4.

5. Pavone P, Spalice A, Polizzi A, Parisi P, Ruggieri M. Ohtahara syndrome with emphasis on recent genetic discovery. Brain Dev 2012;34:459-68.

6. Kodera H, Ohba C, Kato M, Maeda T, Araki K, Tajima D, et al. De novo GABRA1 mutations in ohtahara and West syndromes. Epilepsia 2016;57:566-73.

7. Allen AS, Berkovic SF, Cossette P, Delanty N, Dlugos D, Eichler EE, et al. De novo mutations in epileptic encephalopathies. Nature 2013;501:217-21.

8. Ohno M, Shimotsuji Y, Abe J, Shimada M, Tamiya H. Zonisamidetreatment of early infantile epileptic encephalopathy. Pediatr Neurol 2000;23:341e-4.

9. Malik SI, Galliani CA, Hernandez AW, Donahue DJ. Epilepsy surgery for early infantile epileptic encephalopathy (ohtahara syndrome). J Child Neurol 2013;28:1607-17.

\section{Funding: None; Conflicts of Interest: None Stated.}

How to cite this article: Bhelo $M$, Jagwani $H$, Mukherjee $S$, De $H$, Ghosh A. GABRB3 mutation as a cause of Ohtahara syndrome: A case report. Indian J Case Reports. 2021;7(11):480-481. 